Classification

Physics Abstracts

$68.35 \mathrm{Bs}-61.16 \mathrm{Ch}$

\title{
Microscopie à effet tunnel des surfaces de GaSe hétéroépitaxié sur Si
}

\author{
Guy Michel Guichar, Mohammed Said Belkaid, Marc Morand et Youri Koudinov
}

Laboratoire de Physique des Solides, associé au CNRS $n^{\circ} 154$, Université Pierre et Marie Curie, Tour 13-E2, 4 Place Jussieu 75252 Paris Cedex 05, France

(Reçu le 4 juillet ; accepté le 5 décembre, 1994)

\begin{abstract}
Résumé. - Des surfaces du composé lamellaire GaSe ont été étudiées par microscopie à effet tunnel (S.T.M.). Le montage expérimental que nous avons construit est tout d'abord brièvement décrit. Les images tunnel montrent que ces couches, déposées sur des surfaces de Si(111) par épitaxie par jets moléculaires, sont de bonne qualité, avec quelques défauts localisés. Des images à la résolution atomique sont également présentées.
\end{abstract}

Abstract. - GaSe lamellar compound surfaces have been studied using scanning tunneling microscopy (S.T.M.). First, the experimental set-up which have been built is briefly described. S.T.M. images show that the films, deposited by molecular beam epitaxy are nice, except some localised defaults. Images exhibiting atomic resolution are also shown.

\section{Introduction.}

GaSe est un matériau semiconducteur lamellaire qui possède des propriétés optiques intéressantes notamment pour la génération de la seconde harmonique à 1,06 et $10,6 \mu \mathrm{m}$. Il présente une structure en feuillets, un feuillet élémentaire étant constitué de deux plans d'atomes de gallium entre deux plans d'atomes de sélénium, comme il est représenté sur la figure 1. Dans un feuillet, les liaisons interatomiques sont de type iono-covalent et assurent une bonne cohésion du feuillet. Les liaisons entre feuillets sont en revanche faibles, du type forces de van der Waals. On conçoit alors que ces cristaux présentent une grande anisotropie mécanique qui a largement limité leur utilisation.

A la suite des travaux de Koma et collaborateurs [1] montrant que des lamellaires pouvaient croître par une technique de jets moléculaires sur un autre lamellaire malgré un fort désaccord de maille, cette technique d'épitaxie a été utilisée dans notre laboratoire [2] pour la croissance de GaSe sur silicium. Il est évident que le choix d'un tel substrat peut redonner un intérêt nouveau à ce composé en couches minces. Nous présentons ici les premières expériences de microscopie tunnel effectuées sur cette hétéroépitaxie. 

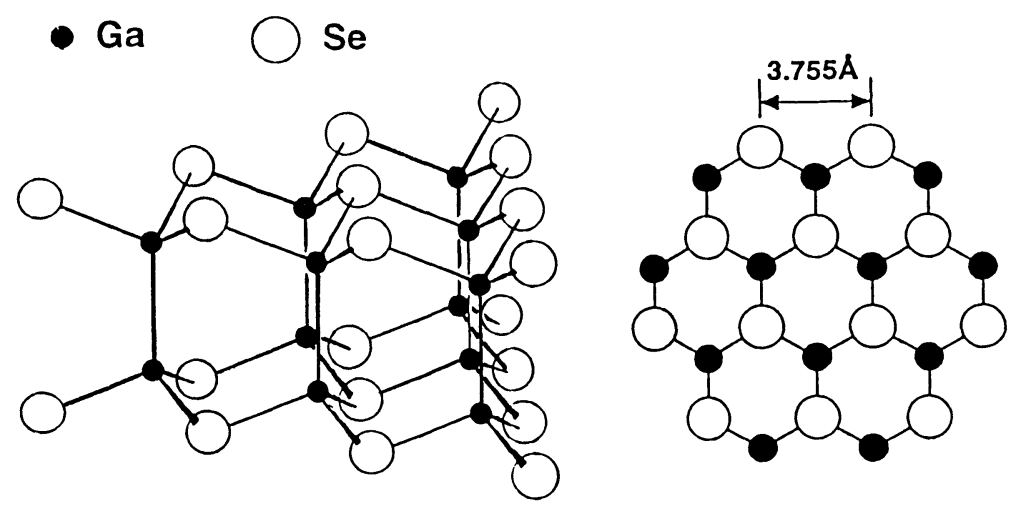

Fig. 1. - Feuillet élémentaire de GaSe : vues en perspective et de dessus.

[GaSe single layer: perspective and top views.]

\section{Montage expérimental.}

Nous avons construit notre microscope à partir d'un module piézoélectrique [3] qui permet d'effectuer d'une part l'approche initiale (translation possible de $25 \mathrm{~mm}$ ), d'autre part le maintien à une distance constante de l'echantillon et le balayage. Cette dernière fonction est assurée par un tube piézoélectrique métallisé sur 4 secteurs séparés. L'application d'une tension électrique sur 2 secteurs diamètralement opposés permet le balayage dans cette direction, tandis qu'une tension appliquée sur tout l'extérieur du tube permet, par une contraction ou un allongement de celui-ci, de maintenir un courant tunnel donné.

Lélectronique de contrôle que nous avons réalisée permet, à partir d'un ordinateur de type PC-AT-386, l'approche de la pointe puis la régulation et le contrôle du courant tunnel, ainsi que l'acquisition du courant tunnel ou de la tension appliquée au tube. L'asservissement est du type P + I, le gain de la boucle ainsi que la constante d'intégration pouvant être ajustés continuement. Le synoptique de l'électronique est reproduit sur la figure 2. Sans rentrer dans un descriptif technique, on peut en donner quelques caractéristiques :

* Fréquence de balayage jusqu'à $30 \mathrm{~Hz}$

* Images jusqu'à $512 \times 512$ points

* Zone maximum explorée : $1 \mu \mathrm{m}^{2}$

* Pilotage informatique complet (balayage,tensions,acquisition...)

* Bruit équivalent en courant : 2 pA dans une bande de $2 \mathrm{kHz}$

Le tube est fixé sur une platine en acier inoxydable, suspendue par 4 ressorts, un amortissement magnétique par courants de Foucault étant assuré. L'ensemble est solidaire d'une bride ultravide de $200 \mathrm{~mm}$ de diamètre. Les pointes sont en tungstène, préparées par attaque chimique. Elles peuvent être régénérées dans l'enceinte ultravide par bombardement électronique et effet de champ. Un outil accessible de l'extérieur permet le changement de pointe entre l'extrémité du microscope et un réservoir contenant jusqu'à 7 pointes. Le transfert d'un échantillon peut également s'effectuer entre la chambre du microscope et un bâti mobile annexe d'introduction et de chargement, l'ensemble étant sous ultravide ( $p=10^{-10}$ Torr).

Un logiciel d'exploitation et de traitement des images a également été développé. Il permet d'effectuer par exemple les mesures de distance, de visualiser les profils et de filtrer les images par transformée de Fourier. 


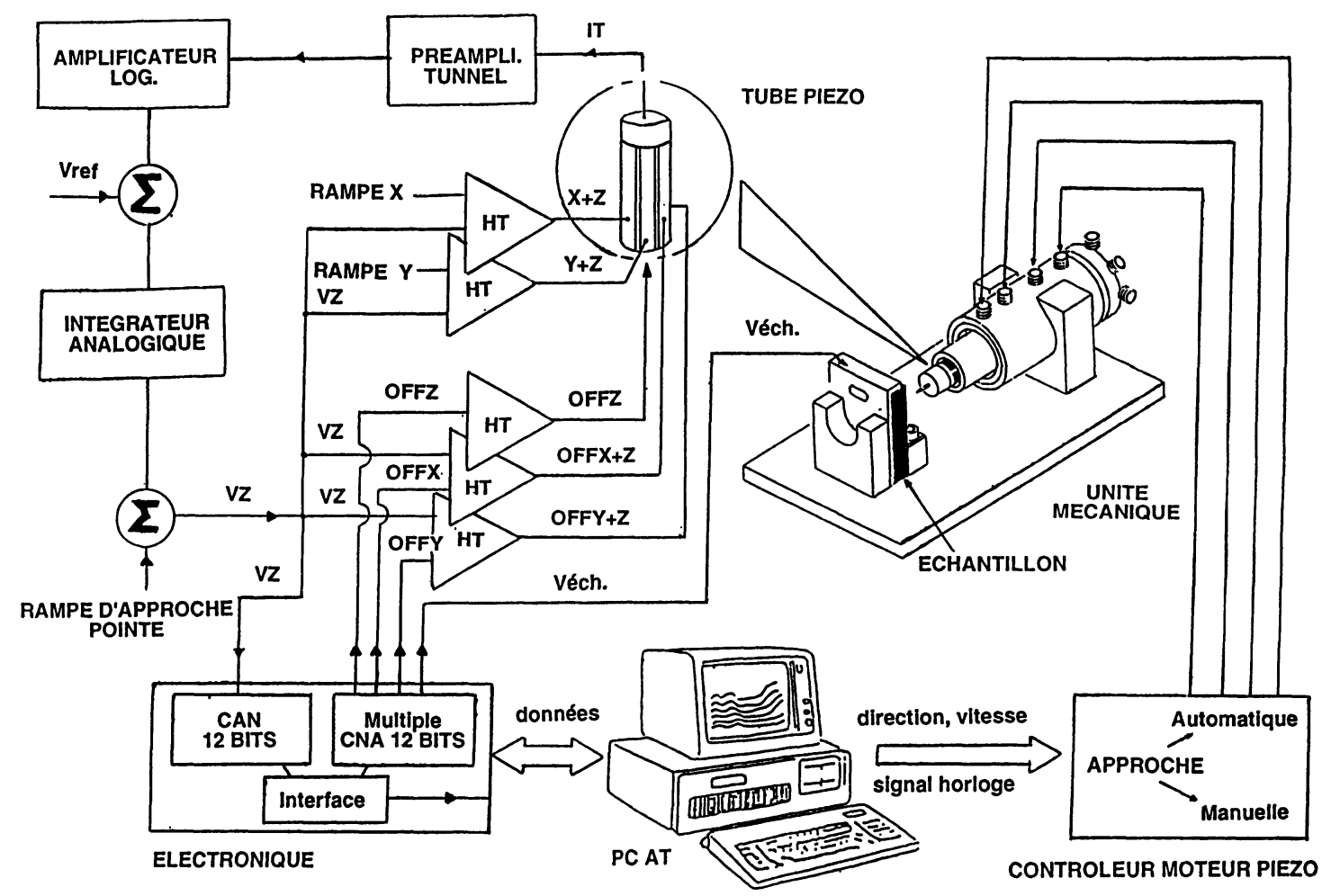

Fig. 2. - Synoptique de l'électronique de contrôle.

[Controlling electronics block diagram.]

\section{Résultats, discussion.}

Les couches de GaSe ont été préparées par épitaxie par jets moléculaires des éléments Ga et Se, les substrats étant des plaquettes de silicium (111). Les détails de cette hétéroépitaxie ont été rapportés par ailleurs [2]. Outre les conditions de température du substrat et de rapport de flux des éléments, le facteur primordial pour l'obtention de couches stoechiométriques de bonne qualité est la préparation de surface. Il est essentiel d'obtenir une surface de silicium aussi ordonnée que possible pour avoir ensuite une croissance à 2 dimensions des feuillets du lamellaire. Le suivi par RHEED au cours de l'épitaxie, aussi bien que les clichés de microscopie électronique en transmission, montrent [4] qu'il en est ainsi pour la surface $\mathrm{Si}(111)$ reconstruite $7 \times 7$ ou $\sqrt{3} \times \sqrt{3} R 30^{\circ}$ obtenue par depôt d'un tiers de monocouche de Ga, et également pour cette même face passivée par l'hydrogène, qui présente alors une structure $1 \times 1$. Sur ces surfaces, le plan (0001) de GaSe (plan du feuillet) est alors parallèle au plan (111) du silicium.

La microscopie à effet tunnel permet de préciser la morphologie de surface des couches épitaxiées. L'image de la figure 3 est caractéristique de nos observations. Elle correspond à une surface de $4000 \times 2000 \AA^{2}$ de GaSe (épaisseur nominale $150 \AA$ ) épitaxié sur $\operatorname{Si}(111) 7 \times 7$. On y distingue des plans, avec très peu de défauts, et également des régions très perturbées. L'analyse du profil des coupes montre qu'on a en fait des terrasses, dont la longueur peut atteindre $1000 \AA$, séparées par des marches dont la hauteur est voisine de $8 \AA$. Ceci correspond à l'épaisseur d'un feuillet (distance entre deux Se équivalents de deux feuillets successifs) qui vaut 7,97 $\AA$ [5].

Des “bosses" de forme pseudo-sphérique existent également. Sur cette image, on peut en voir 


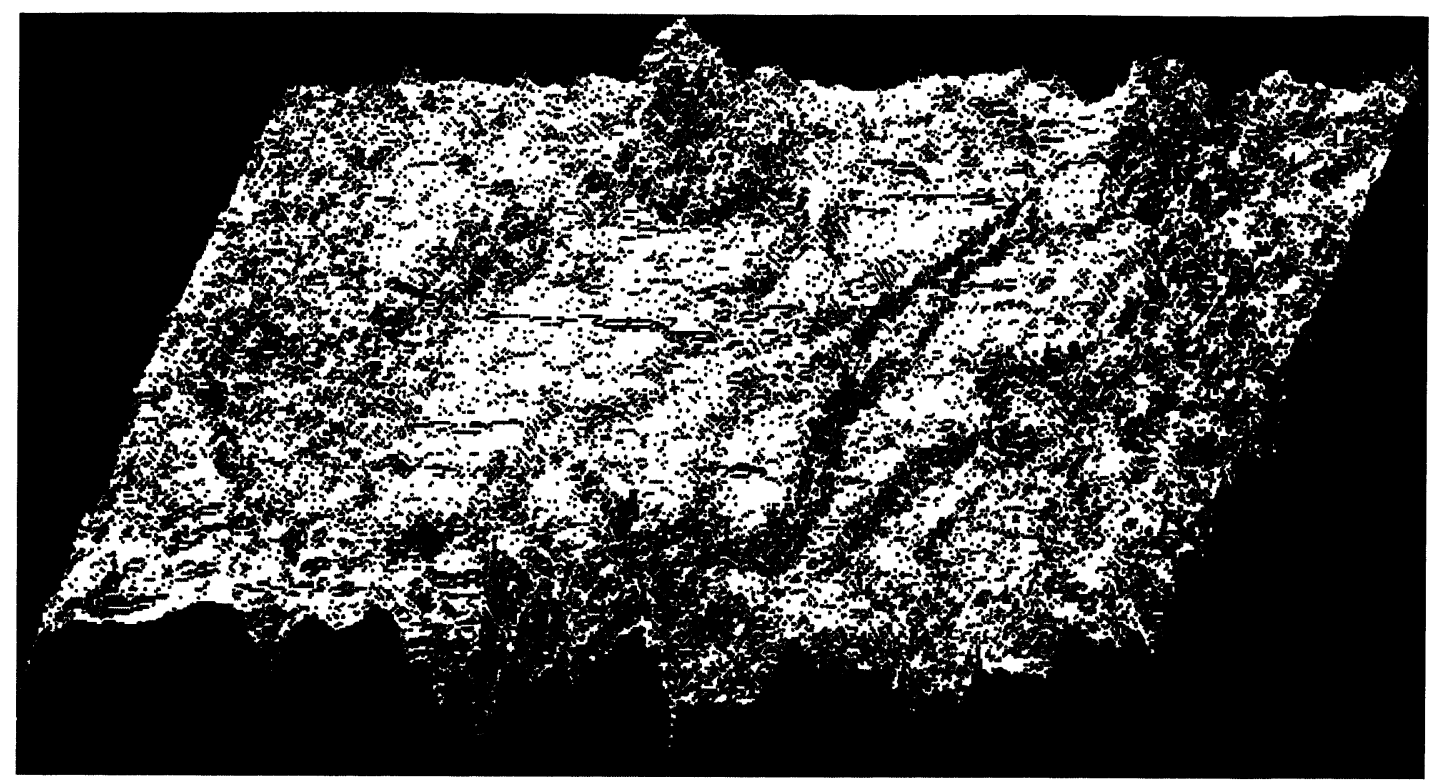

Fig. 3. - Représentation axonométrique d'une surface de GaSe épitaxié sur $\operatorname{Si}(111) 7 \times 7$. Aire balayée : $4000 \times 2000 \AA^{2}$. Variation en hauteur : $400 \AA$.

[Axonometric view of a GaSe on $\operatorname{Si}(111) 7 \times 7$ surface. Scanned area: $4000 \times 2000 \AA^{2}$. Height variation: $400 \AA$.

une d'environ $250 \AA$ de haut et qui s'étend sur près de $250 \times 300 \AA^{2}$. L'analyse de la fluorescence $X$, faite au microscope à balayage, montre qu'il s'agit le plus souvent d'amas riches en sélénium ; quelques amas de gallium pratiquement pur étant également quelquefois observés.

Sur les terrasses peu perturbées, les images de plus petite taille permettent d'observer la maille de surface. L'image de la figure 4, d'environ $35 \times 20 \AA^{2}$, a été prise à “distance constante". Dans ce mode de fonctionnement, on enregistre l'intensité du courant tunnel qui varie ici de 130 à 400 $\mathrm{pA}$, la polarisation étant de $-1,7 \mathrm{~V}$ sur l'échantillon. On retrouve bien la symétrie attendue, la distance entre atomes valant, à l'incertitude d'étalonnage près, 3,7 $\AA$. Cependant, en comparant avec la figure 1, on voit qu'on ne détecte qu'un seul type d'atomes. A priori, on peut penser que ce sont les atomes les plus extérieurs du feuillet, en l'occurence les atomes de sélénium. En effet,alors que la distance entre les deux plans de sélénium vaut $4,77 \AA$, celle entre un plan de gallium et un plan de sélénium est de l'ordre de $1,19 \AA$, cette distance pouvant varier de quelques pour cent suivant le polytype [5].

Cette interprétation est corroborée par le fait qu'avec la polarisation utilisée $(-1,7 \mathrm{~V}$ sur l'échantillon), le courant tunnel est dû à l'émission d'électrons à partir des états occupés au voisinage du sommet de la bande de valence. Les calculs de structure de bandes et de densité de charge électronique [6] montrent que ces états sont essentiellement de caractère $p_{z}$ (dirigés suivant la normale au plan du feuillet) et localisés sur les atomes de sélénium. Sur les atomes de gallium, ces mêmes densités de charge sont très faibles.

Des images à “courant constant" ont également été obtenues. Les figures 5 et 6 en sont des exemples. Sur la première image, de dimensions $50 \times 25 \AA^{2}$, on note dans le coin supérieur droit une région où la résolution atomique est difficilement visible. Lanalyse du profil des coupes montre qu'il s'agit en fait d'un feuillet supplémentaire, sa hauteur moyenne étant de $8 \AA$. Il faut 


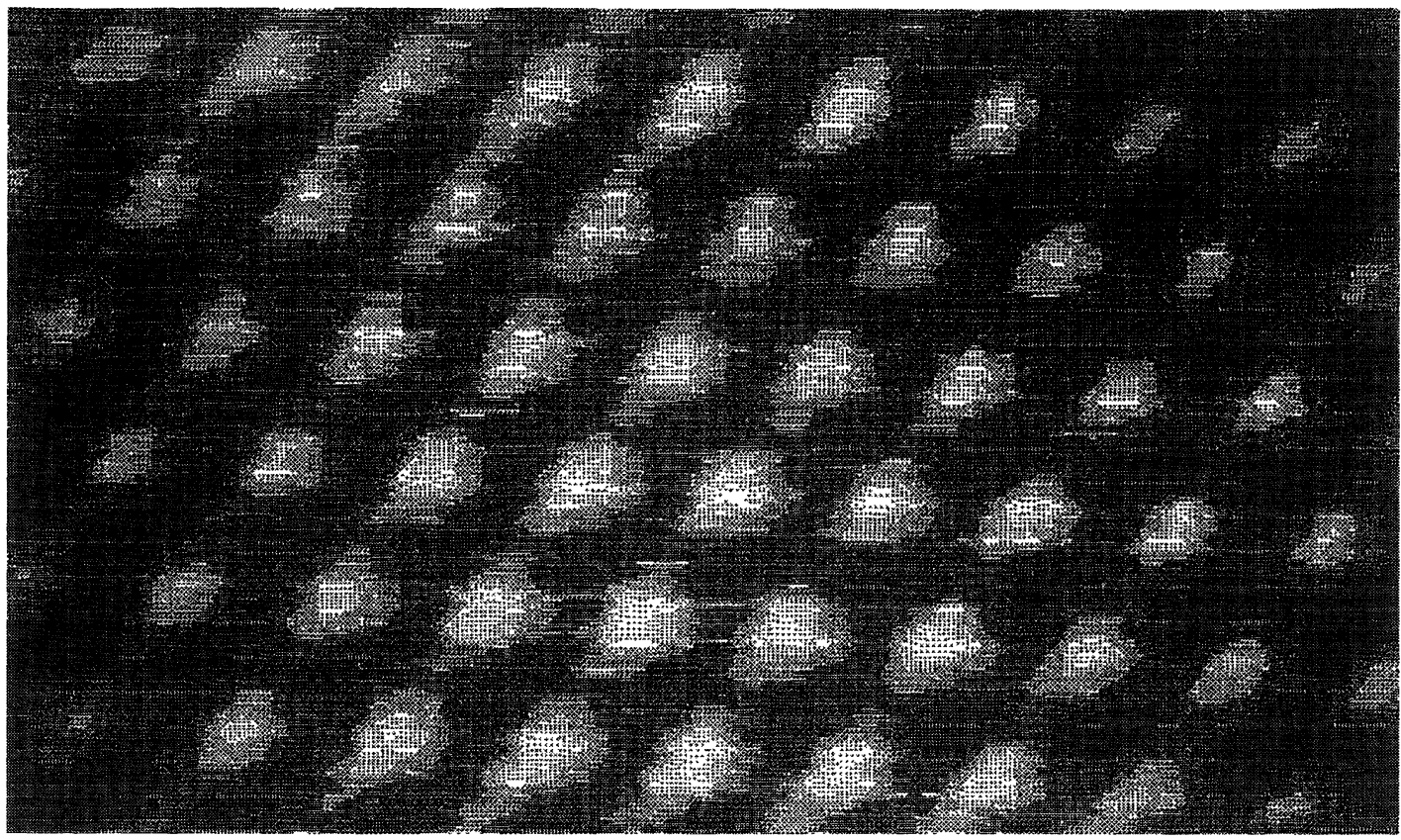

Fig. 4. - Vue de dessus d'une surface de GaSe épitaxié sur Si(111) $7 \times 7$ (mode hauteur constante). Aire balayée : $35 \times 20 \AA^{2}$. Du noir au blanc, le courant tunnel varie de 130 à 400 pA.

[Top view of a GaSe on Si(111) $7 \times 7$ surface (constant height mode). Scanned area: $35 \times 20 \AA^{2}$. The tunneling current varies from 130 to $400 \mathrm{pA}$ from black to white respectively.]

également noter que la modulation "atomique" est importante : de l'ordre de 5 à $6 \AA$, donc bien supérieure à celle attendue. De tels effets sont connus dans d'autres corps lamellaires tels que le graphite. L'explication généralement retenue est une alternance d'attraction-répulsion entre la pointe et les atomes de surface qui amplifie notablement la modulation atomique réelle [7]. Ces effets sont d'autant plus marqués que le rayon de courbure de l'extrémité de la pointe est grand.

La figure 6 correspond à une surface plus étendue : $100 \times 50 \AA^{2}$. On y distingue aussi 2 feuillets. Les régions correspondant au feuillet supérieur sont de l'ordre de $15 \times 15 \AA^{2}$. Les atomes de bord de marche sont plus proches du feuillet inférieur que ceux du centre de ces zones. On a apparemment des morceaux de feuillets légérement en forme de dôme. En l'absence de calculs précis, on peut cependant remarquer que les atomes au bord d'une marche (peut-être des Se) ont une liaison Se-Ga rompue. Il peut s'ensuivre un renforcement de la liaison restante Se-Ga ou une liaison plus forte avec le feuillet inférieur. Dans les deux cas,on pourrait alors avoir une distorsion du feuillet en bord de marche.

Ces différentes images ont toutes été obtenues avec l'échantillon polarisé négativement $(-1,7 \mathrm{~V})$. Comme indiqué précedemment,on sonde alors les états électroniques occupés au voisinage du sommet de bande de valence et le courant tunnel est dû aux électrons émis par la surface de GaSe.

Qu'en est-il des états électroniques vides? les calculs prévoient, sur les atomes de gallium, des densités de charge électronique notables correspondant aux états du bas de la bande de conduction. Compte-tenu de la valeur de la bande interdite (environ 2,2 eV), en polarisant cette fois positivement l'échantillon avec une tension de l'ordre de $1 \mathrm{~V}$, on devrait obtenir une image tunnel de ces atomes. Nos tentatives dans cette polarisation ont été infructueuses. En 


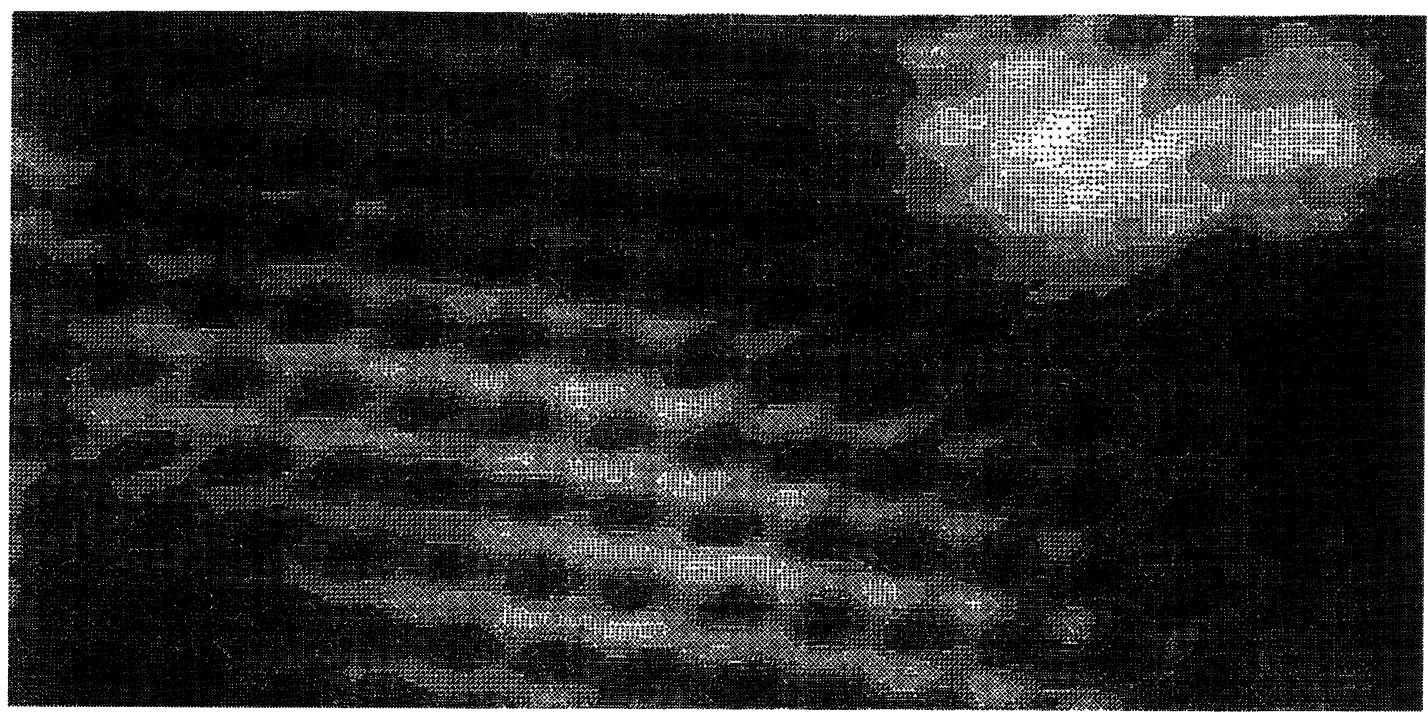

Fig. 5. - Vue de dessus d'une surface de GaSe épitaxié sur Si(111) $7 \times 7$ (mode courant constant). Aire balayée : $50 \times 25 \AA^{2}$. Variation en hauteur : $14 \AA$.

[Top view of a GaSe on $\mathrm{Si}(111) 7 \times 7$ surface (constant current mode). Scanned area: $50 \times 25 \AA^{2}$. Height variation: $14 \AA$.]

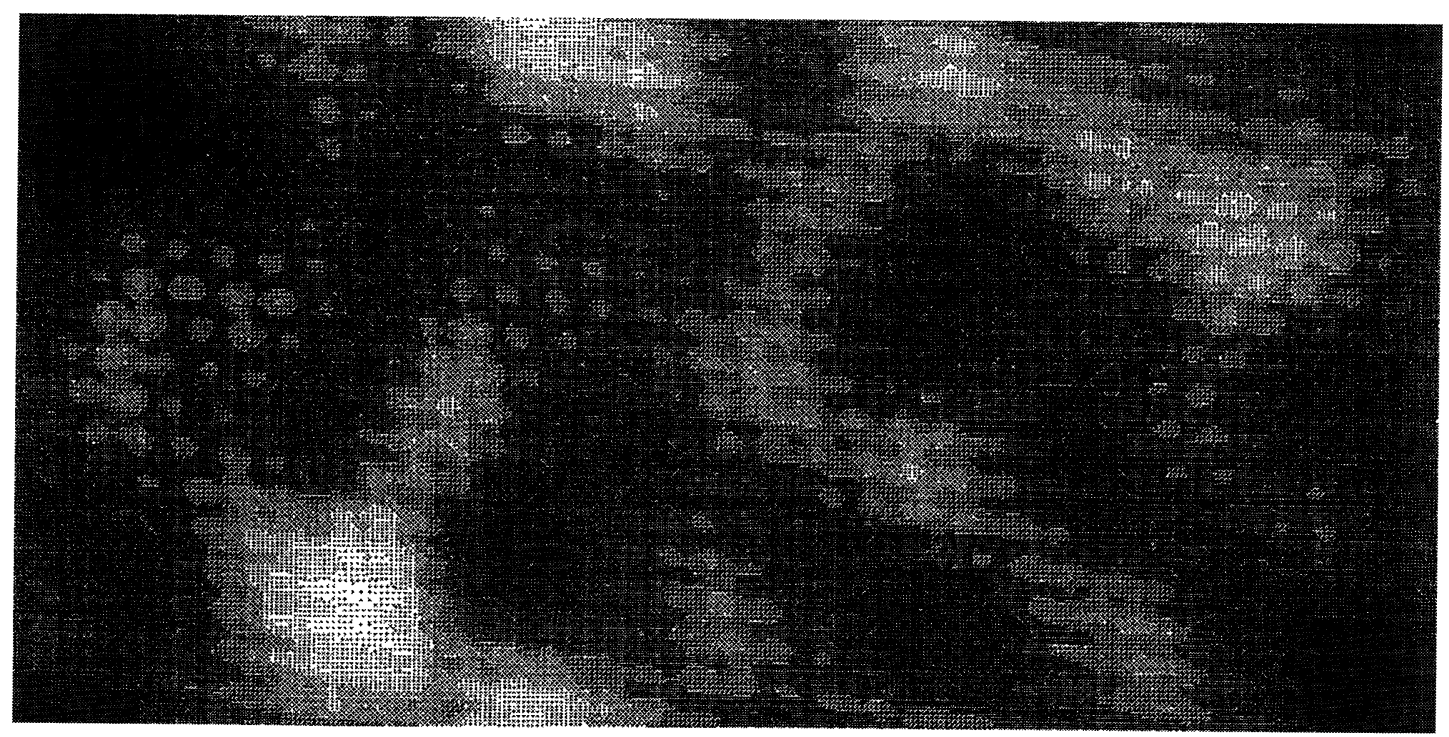

Fig. 6. - Vue de dessus d'une surface de GaSe épitaxié sur Si(111) $7 \times 7$ (mode courant constant). Aire balayée : $100 \times 50 \AA^{2}$. Variation en hauteur : $15 \AA$.

[Top view of a GaSe on $\mathrm{Si}(111) 7 \times 7$ surface (constant current mode). Scanned area: $100 \times 50 \AA^{2}$. Height variation: $15 \AA$.] 
général, avec une tension positive (entre 0,5 et $10 \mathrm{~V}$ ), il nous est impossible d'obtenir un courant tunnel même faible ; la pointe avance vers l'échantillon de plusieurs centaines d'Å sans y parvenir. Quelquefois, on peut obtenir un courant tunnel faible $(<100 \mathrm{pA})$, mais l'image ne présente aucune modulation atomique.

On peut tenter quelque explications sur l'origine de ce comportement asymétrique. La première est liée au fait que la conductivité électrique perpendiculaire au plan des feuillets est très faible dans ces composés lamellaires. Les contacts électriques étant sur la face arrière du substrat silicium, il est sans doute plus difficile d'y collecter des électrons (polarisation positive sur l'échantillon) que d'en émettre à partir de la surface (polarisation négative). Il est également possible qu'il y ait une accumulation en surface de charges négatives qui s'oppose au passage des électrons émis par la pointe. D'autres expériences seront nécessaires pour être plus affirmatif. On pourrait d'une part, prendre des contacts directement sur la surface de GaSe, d'autre part, réduire une éventuelle courbure des bandes en surface en éclairant l'échantillon avec un rayonnement d'énergie supérieure à celle de la bande interdite [8].

\section{Conclusion.}

Nos expériences de microscopie tunnel montrent que l'hétéroépitaxie d'un semiconducteur lamellaire tel que GaSe sur un semiconducteur cubique comme le silicium est réalisable et donne un matériau de bonne qualité. Des images montrant la résolution atomique ont été obtenues, le courant tunnel provenant de l'émission d'électrons à partir de la surface de GaSe. Pour expliquer l'impossibilité d'obtenir des images à partir d'électrons émis par la pointe, il est suggéré qu'il peut exister, en surface de GaSe, une accumulation de charges négatives. Ces expériences seront poursuivies et on s'efforcera notamment d'étudier des couches ultra-minces, d'épaisseur de l'ordre d'un feuillet, pour étudier comment un composé lamellaire peut se lier à un corps tel que le silicium sur lequel existent des liaisons rompues en surface.

\section{Bibliographie}

[1] Koma A., Sunouchi K. and Miyajima T., J. Vacuum Sci. Technol. B3 (1985) 724.

[2] Le Thanh V., Eddrief M., Sébenne C., Sacuto A., Balkanski M., J. Crystal Growth 135 (1994).

[3] Burleigh Instruments, modèle ARIS 11.

[4] Eddrief M., Le Thanh V., Guichar G.M., Belkaid M.S., Zheng Y.L., Gandais M., Bénisty H., Sébenne C., Pétroff J.F., Journées Surfaces et Interfaces, Nancy, 27-28 janvier 1994 (non publié).

[5] Schubert W., Dörre E., Kluge M., Z. Metallk 46 (1955) 216.

[6] Depeursinge Y., Nuovo Cimento 64B (1981) 111.

[7] Soler J.M., Baro A.M., Garcia N. and Rohrer H., Phys. Rev. Lett. 57 (1986 ) 444.

[8] Salvan F., Dumas P., communication privée. 